\title{
SHOCK COMPACTION OF BIOCERAMIC COMPOSITES
}

\author{
M. Stuivinga ${ }^{a}$ and E.P. Carton ${ }^{\mathrm{a}}$, and J. R. de Wijn ${ }^{\mathrm{b}}$ \\ ${ }^{a}$ TNO Prins Maurits laboratory, P.O. Box 45, Rijswijk, The Netherlands
}

${ }^{\mathrm{b}}$ IsoTis B.V, Bilthoven, The Netherlands.

A method was developed for making dense hydroxyapatite-polymer composites. Hydroxyapatite (HA) is a type of calcium phosphate, which is a bioactive material. The polymer used in this work was Polyactive $\mathrm{e}^{\mathrm{TM}} 60 / 40$, a block copolymer from polyethylene oxide (PEO) and polybutylene terephtalate (PBT) in a weight ratio of 60/40. It is a biodegradable polymer with bone bonding properties. In this study the submicron HA starting powders were coated in such a way that a chemical bonding existed with the polymer.

Polymer composites with a high fraction of HA (70-80 wt \%) are of interest as a temporary replacement for bone, but are hard to densify by conventional means. Here it is shown that they can be densified up to about $87-$ $88 \%$ of the theoretical maximum density. When this is followed by a heat treatment in which the polymer chains are allowed to entangle, a sound compact will result. The compact thus obtained is stable in water, contrary to those samples that do not receive a heat treatment.

Furthermore, the limitations in strength that can be obtained for such composites are being addressed here. These findings can be generalized for the densification of polymer (composites) by shock densification means.

\section{INTRODUCTION}

Hydroxyapatite (HA) is an apatite crystal form of calcium phosphate [1], which is similar to the mineral part of human bone and teeth. It has excellent biocompatibility, since it can form a direct chemical bond with hard tissues. As such it is one of the main components in man-made implant materials. However, as an implant for load-bearing applications it is too brittle when used in its pure form. For those applications, metallic materials are currently used. Metallic implants can however give mechanical problems, since the metals used have a much larger stiffness than bone. This may lead to stress shielding and subsequent resorption of the underlying bone. As temporary fixation pins, metallic implants will have to be removed, which requires a second operation, which is a drawback. In general polymers have a smaller stiffness and strength than bone, but they can be engineered to dissolve in the body, which is interesting for the purpose of biodegradable materials. For instance, bone pins should only give strength when needed, but preferentially should resorb in the body, when the bone tissue has healed.
In a large research effort at the Biomaterials group of the University of Leiden and IsoTis B.V. special polymers were designed which are both biodegradable and have bone bonding properties. The polymer is called Polyactive ${ }^{\mathrm{TM}}$ 60/40, (PA) and consists of a block co-polymer from polyethylene oxide (PEO) and polybutylene terephtalate (PBT) in a weight ratio of $60 / 40[2,3]$.

Looking at natural bone it seems advantageous to use a composite material consisting of a combination of a high fraction of HA (70-80 wt.\%) and a polymer in order to obtain better mechanical properties, such as an elasticity modulus closely resembling that of bone.

Mixing homogeneously high volume fractions of a ceramic into a polymer presents a problem. In the present work this was solved by a special coating technique, called grafting, in which the PA is at one side chemically coupled to a submicron HA particle, as described in Section 2. This coating was shown to improve the mechanical properties of HAPA composites in the wet state for small HA filler ratios (< 30-vol.\%) [3]. For a higher ceramics fraction ( $>50$-vol.\%), compaction of HA-PA composite materials was found to be impossible by 
standard hot-pressing techniques. Therefore, it was decided to investigate the use of shock compaction.

For polymers high strain rates, as occurring during dynamic compaction will lead to increased brittleness, making crack free densification harder. In fact, fracture surfaces of shock compacted pure PMMA grains $(1-50 \mu \mathrm{m})$ had shown interparticle cracking, which indicated that the bonding between particles was weak [4]. Moreover, dynamic compaction of powder mixtures consisting of HA (50-90 vol.\%) and PMMA had shown the possibility of obtaining dense composites with conservation of the polymer properties [5]. But the question remained if shock compaction would lead to bonding, though the results of Blazynski et al. on silica-PVC mixtures were pointing in that direction $[6,7]$.

To investigate this further for the composites of interest, a series of experiments was set up on HAPA (10-wt \%) materials and also for comparison on some other HA-polymer (10-30-wt. \%) composites. Here, the aim was to obtain dense composites with bone resembling stiffness values and than to test their bonding strength by bending measurements.

\section{STARTING MATERIALS}

HA powder was obtained from Merck (Darmstadt, Germany) as a fluffy powder with a BET specific surface of $60 \mathrm{~m}^{2} / \mathrm{g}$, see Figures 1 and 2. The powder was dried in vacuum at $60^{\circ} \mathrm{C}$ before grafting. The process of grafting polymers to the surface is described in [8]. Briefly, a coupling agent was used to bond the surface hydroxyl of HA with polymer. In the case of poly-(methylmethacrylate) (PMMA) and poly-(butylmethacrylate) (PBMA) trimethoxy-methacryloxy-propylsilane (TMPTS) was used to couple the PMMA and PBMA to the HA surface. In the case of Polyactive 60/40 hexamethylene diisocyanate (HMDI) was used as a bridge between the hydroxyl groups of HA and those on the chain ends of the polymer. Unbound polymer was removed by treating the resulting powders with several rinses of solvent. The amount of grafted polymer was determined by thermal gravimetric analysis (TGA).

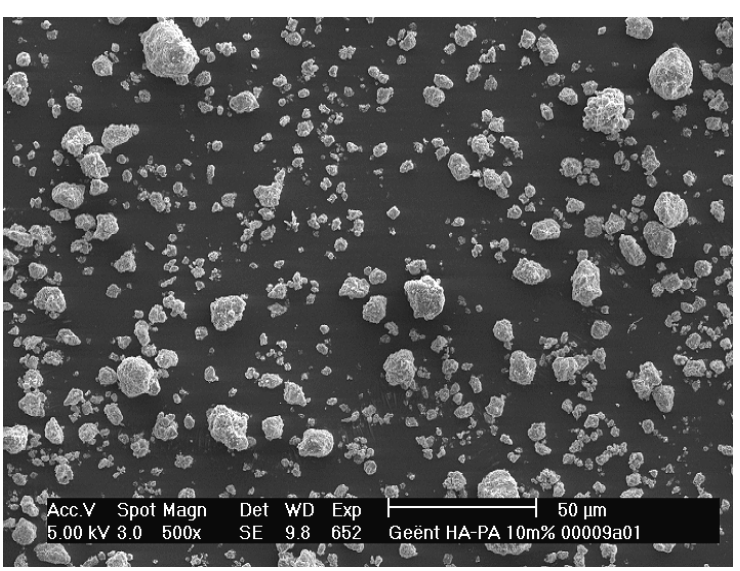

Figure 1. Grafted HA-PA (10 wt \%) powder

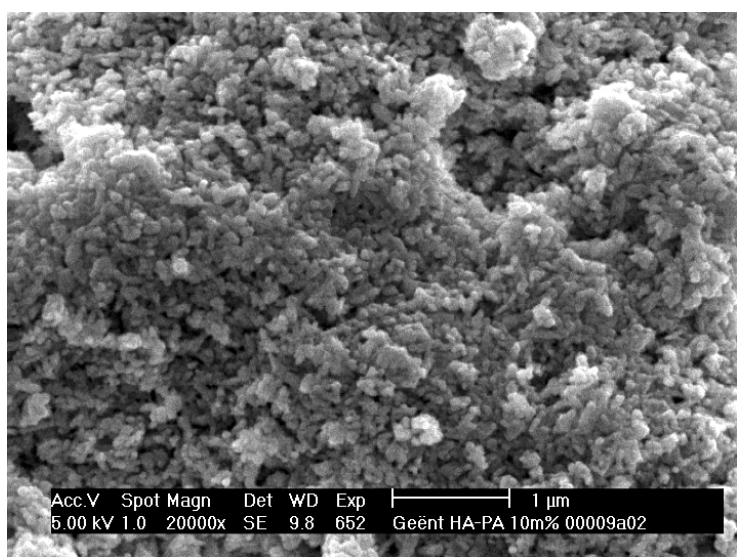

Figure 2. Grafted HA-PA (10 wt \%) powder. Image was taken inside one agglommerated grain and shows the primary grains inside one grain.

\section{EXPERIMENTAL ARRANGEMENT}

\subsection{Explosive compaction}

Explosive compaction was done in the direct cylindrical configuration. The experiments are listed by number (nr.) in Table I.

In the first experiment (nr. 252) the grafted HAPMMA (30 wt\%) powder was uni-axially compressed at $300 \mathrm{MPa}$ in an aluminum tube with an inner diameter, $D_{i}$ of $9.5 \mathrm{~mm}$, an outer diameter of $14 \mathrm{~mm}$ and a length of $100 \mathrm{~mm}$. 
Table I.

Compaction, Young 's modulus $(\mathrm{E})$ and hardness $\left(\mathrm{H}_{\mathrm{v}}\right)$ data. Here $\mathrm{T}$ is the temperature of heat treated samples.

\begin{tabular}{|c|c|c|c|c|c|c|c|c|c|c|c|}
\hline Exp & Material & Polymer & HA & $\mathbf{D}_{\mathbf{i}}$ & $\rho_{i}$ & $\rho_{\mathrm{f}}$ & ex. & $\mathbf{d}_{\mathrm{ex}}$ & $\mathbf{T}$ & $\mathbf{E}$ & $\mathbf{H}_{\mathbf{V}}$ \\
\hline nr. & & wt\% & vol- \% & $(\mathbf{m m})$ & $\% \mathrm{TMD}$ & $\% \mathrm{TMD}$ & & $(\mathbf{m m})$ & ${ }^{\circ} \mathbf{C}$ & (GPa) & (GPa) \\
\hline & $\mathrm{HA}$ & & & & & & & & & 111 & 4.6 \\
\hline & PMMA & & & & & & & & & 5.4 & 0.17 \\
\hline & PA & & & & & & & & & $3.7 \times 10-3$ & \\
\hline 413 & HA-PA & 10 & & 20 & $66(\mathrm{CIP})$ & 87 & WPB & 22 & 200 & & \\
\hline 414 & HA-PA & 10 & 77 & 17 & $66(\mathrm{CIP})$ & 88 & WPB & 22 & 200 & & 0.81 \\
\hline 415 & HA-PA & 10 & & & $66(\mathrm{CIP})$ & 90 & WPB & 22 & 200 & & \\
\hline 381 & HA-PA & 10 & & 9.5 & 54 & 91 & WPB & 16 & 150 & & \\
\hline 382 & HA-PA & 10 & & 9.5 & 55 & 100 & WPB & 17 & 200 & & \\
\hline 333 & HA-PA & 10 & 77 & 20 & $70(\mathrm{CIP})$ & 87 & WPB & 18 & & 32 & 0.86 \\
\hline 333 & HA-PA wet & 10 & 77 & 20 & $70(\mathrm{CIP})$ & 87 & WPB & 18 & & 24 & 0.49 \\
\hline 309 & НА-РВМА & 20 & 60 & 9.5 & 89 & 108 & AMPA & 14 & & 15 & 0.5 \\
\hline 252 & HA-PMMA & 30 & 53 & 9.4 & 85 & 94 & AMPA & 16 & & & \\
\hline 310 & HA-PMMA & 30 & 53 & 30 & 80 & 100 & AMPA & 21 & & 20 & 0.46 \\
\hline 334 & HA-PMMA & 30 & 53 & 17 & $85(\mathrm{CIP})$ & 95 & WPB & 17 & & 18 & 0.46 \\
\hline
\end{tabular}

The tube was filled by compressing several powder batches. In this way the powder obtained a starting density, $\rho_{\mathrm{i}}$, of $85 \%$ TMD (Theoretical Maximum Density). This density was calculated using the mass fractions of HA and PMMA which were determined by TGA, in combination with the specific density of the components, 3.16 and 1.2 $\mathrm{g} / \mathrm{cm}^{3}$ respectively.

The aluminum tube was closed by metal plugs and centered in a PVC-tube with an inner diameter of $48 \mathrm{~mm}$ and a length of $174 \mathrm{~mm}$. The space between both tubes was filled with an $17 \mathrm{~mm}$ thick layer, $\mathrm{d}_{\mathrm{ex}}$, of an ammoniumnitrate based powder explosive called AMPA. This is a PML melange with a detonation velocity of $3.6 \mathrm{~km} / \mathrm{s}$. From the reduction of the inner diameter of the aluminium tube the density of the powder after shock compaction was calculated to be $94 \%$ TMD.

A sample of HA-PBMA(20 wt\%) was shock compacted in a similar way, in an aluminium tube with a diameter of $20 \mathrm{~mm}$ (nr. 309). Some transverse cracks could be seen in this compact, after the aluminium tube was removed by sawing. The number of transverse cracks found was about equal to the number of compressed powder batches. Therefore, the reason of these cracks was sought in an inhomogeneous density distribution caused by the batchwise precompaction. This was verified by radiography of aluminum tubes that were filled in the same way. By this technique it became clear that there existed in fact a difference in density between the top of a powder batch and the bottom of the next batch.

Uniaxial compression of the HA-PMMA (30 $\mathrm{wt} \%$ ) powder in the following experiment (nr. 310) was performed in one step in a special die with conical walls The walls were made conical to facilitate the removal of the precompressed powder from this die, which was only $30 \mathrm{~mm}$ long. After compression up to a density of $80 \%$ TMD, the sample was inserted in a different aluminum tube with an inner diameter of $30 \mathrm{~mm}$ and a length of 
$120 \mathrm{~mm}$. The compact was sandwiched between loose alumina powder because this would have about the same change in relative density after shock compaction. As a result, the tube contraction is expected to be everywhere about the same. This sample was shock compacted to full density (100\% TMD).

In the following experiments the samples were precompacted by cold isostatic compression (CIP). In this technique the powder is poured in an elastomer tube which is closed by two elastomer plugs. The elastomer tube is submerged in water which is situated in a pressure vessel. By closing the vessel and pressurizing up to $300 \mathrm{MPa}$, the powder will be compacted hydrostatically In this way precompaction takes place in both axial and radial direction in a homogeneous way. This was verified by radiography, that did not show any difference in density in the compact after CIP.

During explosive compaction the sample has to fit tightly in a metal tube. Therefore, the cylindrical sample was taken out of the elastomer and wrapped in a $100 \mu \mathrm{m}$ thick copper foil so that it could fit in a metal tube with a slightly wider diameter than the sample itself. The tube was closed by metal plugs as usual.

This method was used to precompact grafted HA-PMMA (30 wt $\%$ ), nr. 334, and HA-PA (10 wt $\%)$, nr. 333, up to a homogeneous starting density of 85 and $70 \%$ TMD, respectively. After wrapping in copper foil the samples were put in a stainless steel tube of the nearest dimension. Shock compaction was performed with a $18 \mathrm{~mm}$ explosive layer of Wetter Permit-B (WEB) from Dynamit Nobel $\mathrm{GmbH}$, that has a detonation velocity of 2.8 $\mathrm{km} / \mathrm{s}$

After compaction, the walls of the tube were axially sawn from two sides untill the copper foil became visible. Then, by separation of the two tube halves the sample could be released. The copper foil was very difficult to unwrap, due to some local (explosive) welding of the copper foil. The density of the compact was determined by the diameter reduction and also by measuring the dimensions and the weight, see Table 1.

The explosive welding of the copper foil could be avoided by using a Kapton (polyimide) foil on top of the copper foil. By wrapping the sample in this bi-material foil the copper-copper contact and therefore the welding problem was in fact eliminated in samples of the 400 series (413-415).
In Figure 3 one of those samples wrapped in foil is shown.

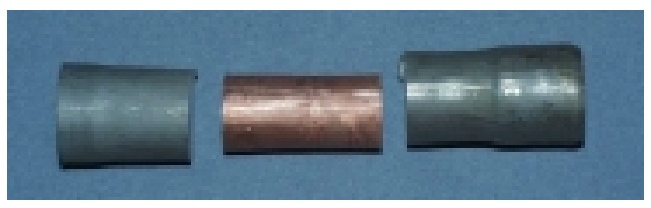

Figure 3. Sample 415 (in the middle), wrapped in copper-Kapton foil, and removed from the stainless steel tube shown besides

\subsection{Stability of HA-PA in water}

During storage in air, the HA-PA samples appeared to fracture slowly. For instance, when a sample was crack-free directly after shock compaction, e.g. nr. 333, it would fragment within three weeks of storage in air. After compaction, this sample was still kept in the metal tube. Then, swelling became apparent which was likely caused by the uptake of humid air. Though the radial expansion of the sample was hindered by the metal containment, the sample was sawn and therefore the top and bottom of the tube were in open contact with air. When by axial swelling the sample was sticking out of the tube, this part could also expand (swell) radially. Subsequently this protuding surface broke off, and a a fresh surface in contact with air was created. In this way a repeating fracture mechanism led to multiple fracturing (layering) of the sample.

When a HA-PA (10 wt \%) sample was immersed in water, fragmentation was much faster and took place in a few minutes This was a further indication that the already known hygroscopical character of the Polyactive was probably responsible for this fracturing.

Concerning the future application as a bone replacing biomaterial, the instability of the HA-PA samples in water forms a real problem. It was first tried to saturate the powder with humidity and to compact that powder explosively. However, the powder turned into mud, so this gave no solution either.

The fast fracturing in water in combination with the relatively low final densities obtained with the HA-PA samples (circa 90\%) seemed to indicate that the permeability for water was the main cause of the problem. Since PA is chemically coupled to the HA-particle surface, fast water transport can ideally only occur between the grafted particles. If 
the PA chains of the grafted particles do not bond during shock compaction, there will remain some open porosity which gives pathways for water. The PA is known to absorb a large fraction of water. The water stability problem can therefore be solved by changing the open porosity into closed porosity. This can be done by giving HA-PA samples a heat treatment after shock compaction, causing the polymer chains to entangle.

This solution was further investigated as follows Two HA-PA samples, nrs. 381 and 382, were heat treated in $\mathrm{Ar}$ for one hour at a temperature of 150 and $200{ }^{\circ} \mathrm{C}$ respectively. These temperatures are respectively below and above the glass temperature of the PBT $\left(195{ }^{\circ} \mathrm{C}\right)$, the stiff component of the PA. The sample heat treated at $150^{\circ} \mathrm{C}$ became stable in air, but still fractured when immersed in water. The sample heat treated in $\mathrm{Ar}$ at $200{ }^{\circ} \mathrm{C}$ remained stable for more than ten weeks immersed in water.

Summarizing these results, the following process route for the fabrication of sound HA-PA (10 wt \%) compacts was obtained:

- precompaction by CIP

- $\quad$ wrapping the sample in copper-Kapton foil

- $\quad$ insertion of the sample in stainless steel tube

- $\quad$ shock compaction with WPB

- heat treatment (one hour) at $200^{\circ}$

\subsection{Measurements}

\section{Hardness}

The hardness of the samples has been measured on flat grinded surfaces using a Vickers microhardness tester. The force and holding time were $5 \mathrm{~N}$ and 20 seconds, respectively.

\section{E-modulus}

The elastic moduli of the samples have been calculated from the measured transversal and longitudinal sound velocities measured at 10 and 2.25 $\mathrm{MHz}$, respectively in combination with the specific density of the samples. The values thus obtained are dynamic moduli and should be checked mechanically to obtain the static moduli.

\section{EXPERIMENTAL RESULTS}

In Table I the compaction results are shown of the polymer coupled HA composites. Here, $D_{i}$ is the inner diameter of the tube containing the powder before compaction, and $T$ is the temperature of the heat treatment, if applicable. The density value for the smallest $D_{i}$ is not very accurate (circa $10 \%)$.

HA-PMMA (30wt \%) and HA-PBMA (20wt \%)

From Table I it can be seen that samples with 30wt \% PMMA corresponding with a vol.- $\%$ HA of $53 \%$ have a density ranging from 94-100\%. Moreover, slices that are a few $\mathrm{mm}$ thick are transparent to visible light, which is a clear indication of a very high density. In Figure 4 the fracture surface of a HA-PBMA compact is shown. Here, on some places a brittle fracture surface of HA could be found with some smaller grains upon it. These small grains are most likely the PBMA coated HA crystallites, having a diameter of about $100 \mathrm{~nm}$. From the TMD values and the SEM image it can be concluded that the sample has a density near theoretical.

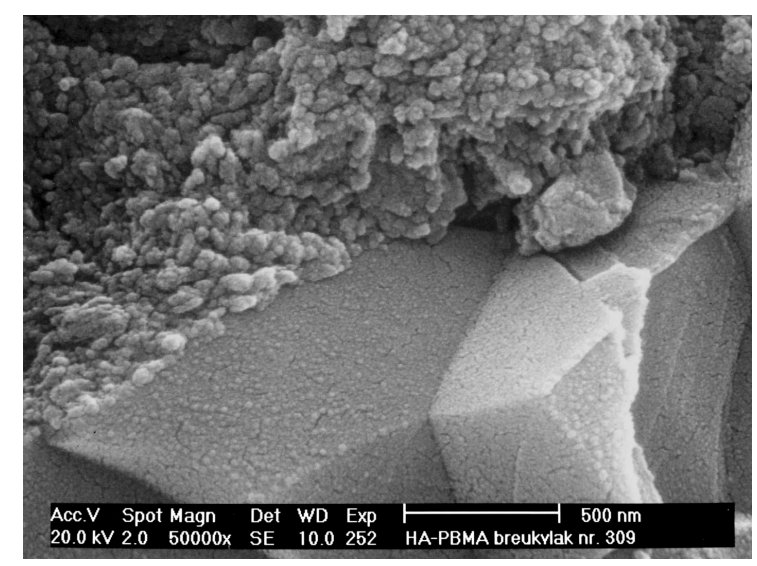

Figure 4. Fracture surface of HA-PBMA.

\section{$H A-P A(10 w t . \%)$}

Samples of the 400 series have been fabricated using the evolved process method described in section 3 . The density was on the average about 90 $\%$ TMD. This is about $10 \%$ lower than that of the former materials, which contain a higher percentage of polymer. In Figure 5 a typical fracture surface of such a compact is shown. On many places individual grains can be recognized, indicating that fracture is mainly intergranular.

As a result of the heat treatment sample 414 could be kept under water for weeks and stayed intact. 


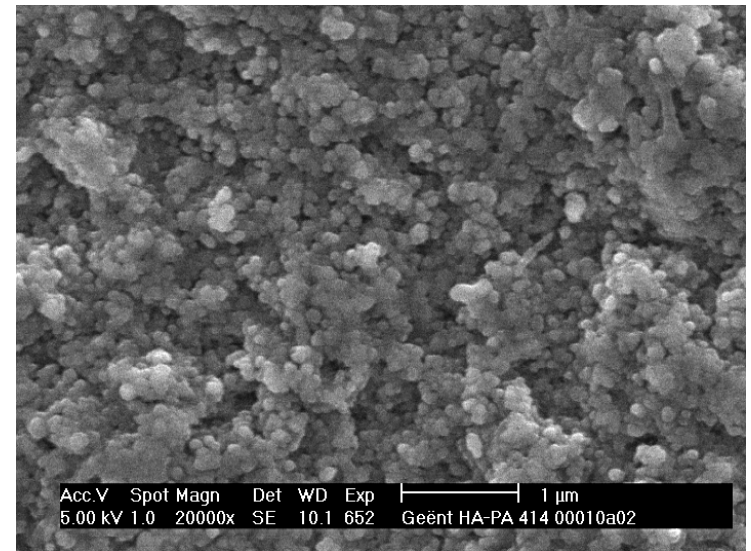

Figure. 5. Fracture surface of HA-PA (10 wt $\%)$

\section{MECHANICAL PROPERTIES}

From ultrasonic measurements and the density, the Young's modulus or stiffness (E) can be determined. Hardness was measured using the Vickers method. Results are presented in Table I.

For HA-PA $(10$ wt. $\%)$ the E-modulus was determined in the dry and wet state. It resulted in a value of respectively 32 and $24 \mathrm{GPa}$. Given the low stiffness of PA ( $3 \mathrm{MPa}), \mathrm{E}$ is mainly determined by the vol.-\% HA. Note that this sample had not undergone a heat treatment, which explains the decrease of $\mathrm{E}$ in the wet state with the corresponding reduction in hardness. The Emodulus for composites with a higher polymer fraction, HA-PBMA and HA-PMMA is lower, respectively $15 \mathrm{GPa}$ and $18-20 \mathrm{GPa}$.

These stiffness values have to be compared with the ones for compact bone that range between 15 and $19 \mathrm{GPa}$. Furthermore, it has to be noted that ultrasonically measured values represent maximum values, which should be compared with values obtained from tensile testing experiments [9].

From sample nr. 334, pieces of $20 \times 10 \times 3 \mathrm{~mm}$ were cut in order to perform three point bending measurements. Measured bending moments were $0.8,3.1$ and $4.6 \mathrm{Nm}$, respectively, which is typically one order of magnitude less than required for fracture plates [1]. The highest value corresponds to a bending strength lower than 5 $\mathrm{MPa}$. Here, the lowest strength values were related to inhomogeneities in the sample such as a $20 \mu \mathrm{m}$ large HA particle in the compact matrix. From the samples of the 400 series, no specimens could be sawn for bending measurements, since they were flaking off which clearly indicates their brittle behavior.

\section{DISCUSSION}

\subsection{Bonding?}

Though the HA-PA (10 wt \%) composites were sound compacts without cracks, which could be immersed in water, their strength was found to be insufficient. This raised the question if bonding had occurred. If the HA is properly coated this bonding should take place between the $P A$ molecules. Bonding can e.g. be affected by the following factors: heat treatment, PA coverage, and density which will be considered next.

\subsection{Heat treatment}

It was established that HA-PA composites which had undergone a heat treatment at $150{ }^{\circ} \mathrm{C}$ fell apart when immersed in water, while those which had undergone a heat treatment at $200{ }^{\circ} \mathrm{C}$, above the glass transition temperature of the PBT component of the polymer $\left(195^{\circ} \mathrm{C}\right)$ stayed intact for at least 24 hrs.

It is known that in order to obtain polymer bonding, time and temperature are required so that the polymers can entangle Apparently, shock densification alone at the present low shock pressures generates insufficient heat and/or for a too small period of time for bonding to occur. This was confirmed in the following control experiment. When pure PA (particle size $250 \mu \mathrm{m}$ ) was shock compacted: no compaction or bonding occurred, but after a heat treatment at $150{ }^{\circ} \mathrm{C}$ the material became rubbery and at $200{ }^{\circ} \mathrm{C}$ melting was shown to occur. This supports the above mentioned results.

\subsection{Polymer coverage}

The PA coverage can be calculated as follows. Given the measured specific surface density (BET method) of the HA particles, $S=60 \mathrm{~m}^{2} / \mathrm{g}$ and the wt- $\%$ of PA, there follows an average PA coverage of only $2 \mathrm{~nm}$ thickness.

\subsection{Density}

The starting powder, shown in Figures 1 and 2 consists of agglomerates of very small uniform spherical particles, with a primary grain size of the order of $100 \mathrm{~nm}$ and with a calculated PA coverage of a few nm. During shock compaction the 


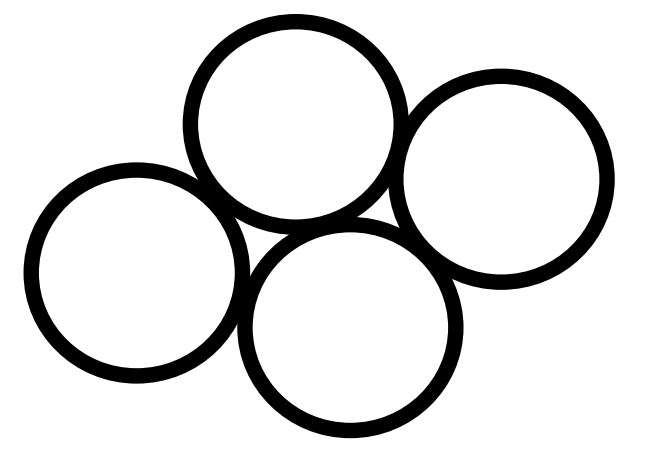

Figure 6. Hard sphere model presentation of HAPA particles after DC and heat treatment. The PA layer is printed in black.

agglomerates will break into the primary grains [10]. Therefore, we may consider these particles as an assembly of small uniform hard spheres. It is known that the theoretical maximal density will then be 74 vol.- $\%$ [11]. Furthermore, from powder technology it is known that the filling degree by uniform particles is ranging between 65-70 vol.- $\%$. [12] The density after compaction is lying between $87-90 \%$ TMD, giving a $10-13$ wt \% of remaining porosity. Since the HA particles are very small, they are considered to be fully dense. The remaining space between the HA could be filled up with 23-28 vol.\% PA (using $\rho_{\mathrm{PA}}=2.1 \mathrm{~g} / \mathrm{cm}^{3}$ ). This calculated pore space is as expected for uniform spheres. Therefore, the conclusion must be that in the present case, the density is about as high as can be expected and that the empty space where the particles meet (see Figure 6) will cause weakness and regions without bonding. This can also explain the brittle behavior found during the sawing of the bend test samples.

\subsection{Strength}

After compaction and a proper heat treatment at a temperature where the polymer chains can entangle, the HA-PA can be submersed in water and stays intact. This indicates that the water cannot penetrate the material as easy as before. Also the results on pure PA do indicate that the polymer has undergone proper temperature conditions to entangle and bond. However the remaining pores in the matrix, see Figure 6, will reduce the strength. Furthermore the small layer thickness of the PA will make load transfer difficult. Theoretically it is expected that the bending strength of a polymer filled up with ceramic particles with an aspect ratio (length to diameter ratio) of about 1 will be determined by the polymer strength [13]. The bending strength of pure $\mathrm{PA}$ is circa $7 \mathrm{MPa}$. By filling a polymer matrix with $\mathrm{HA}$, the E-modulus will increase but the bending strength will decrease. More (directional) strength could be introduced by using fibers in one direction or by using stronger polymers and a higher polymer fraction. Here, a good adhesion between polymer and ceramic is a prerequisite.

Natural bone is made up of nm sized HA fibers in a specially constructed collagen matrix, giving it strength (120-140 MPa). However, from a biomaterials point of view the use of small polymer coated fibers will give unwanted inflammatory reactions (fibrosis).

In this respect it is interesting to go back to the results of the work of Blazynski et al. [6.7], who shock compacted silica-PVC mixtures at different pressures but without further heat treatment. For silica-PVC $(75 \mathrm{v} \%)$ they found an increase in the compression strength of pure PVC from $30 \mathrm{MPa}$ to $55 \mathrm{MPa}$. However, the bending strength was reduced to $12 \mathrm{MPa}$. For comparison: the bending strength for extruded PVC $\left(\rho=1.4 \mathrm{~g} / \mathrm{cm}^{3}\right)$ is found to be 60-70 MPa [14]. This indicates a certain lack of bonding.

The findings of this research can probably be generalized for the shock compaction of polymer composites (and pure polymers). Shock compaction alone will not result in proper bonding but a further heat treatment above the glass transition temperature, where the polymer chains become mobile is necessary to ensure good bonding. Furthermore, it is worth mentioning that the developed chemical coating technique of HA particles could be used similarly for coating silica with a polymer and making products having the hardness of glass, but with increased impact resistance.

\section{CONCLUSIONS.}

A process method is developed for the densification of hydroxyapatite-polymer composites, using shock compaction followed by a heat treatment at a temperature where the polymer chains become mobile. In this way sound compacts with high ceramics fraction have been fabricated, which were stable in water. Because of the remaining porosity the composites were too brittle and lacked strength. The findings in this research supports the view that shock compaction of 
polymer composites will have to be followed by an appropriate heat treatment in order to obtain good bonding between the constituents.

\section{ACKNOWLEDGEMENTS}

The authors would like to thank J. Roosma for his assistance with the experiments. Discussions with J. Olijslager, F. van Hattum, and F. Vercauteren (TNO) are greatly acknowledged.

\section{REFERENCES}

1 J.B. Park en R.S. Lakes, Biomaterials, an introduction, $2^{\text {nd }}$ ed, 1992 (Plenum Press, New York)

2 Q. Liu, J.R. de Wijn, D. Bakker and C.A. van Blitterswijk, Surface modification of hydroxyapatite to introduce interfacial bonding with polyactive ${ }^{\mathrm{TM}} 70 / 30$ in a biodegradable composite, J. Mat. Sc: Mat. in Medicine 7 (1996), 551-557.

3 Q. Liu, "Hydroxyapatite/polymer composites for bone replacement", Thesis, University of Twente, 1997.

4 E.P. Carton, "Dynamic compaction of ceramics and composites", Thesis TUD, 1998, Ch.6.

5 E.P. Carton, M. Hofmeijer, M. Stuivinga and J.R. de Wijn, Proc. $5^{\text {th }}$ European Conference on Adv. Mater. Processes and Applications,
Maastricht, The Netherlands, April 1997 (ed. L.A.J.L. Sarton and H.B. Zeedijk), 2, pp 155158.

6 T.Z. Blazynski, "Explosively consolidated PVC-alumina powder mixtures", J. Mat. Process. Techn., 39 (1993) 389-404.

7 T.Z. Blazynski, "Explosively consolidated PVC-silica powder mixtures", Proc. EXPLOMET, 1995.

8 Q.Liu, J.R. de Wijn, K. De Groot, and C.A. van Blitterswijk, "Surface modification of nano-apatite by grafting organic polymer" Biomaterials 19, 1067-1072, 1998

9 R.S. Gilmore and J.L. Katz, "Elastic properties of apatites”, J. Mat. Sci. 17 (1962), 1131-1141.

10 From previous shock compaction results on zirconia powders.

11 C. Kittel, Introduction to Solid State Physics, 4 ed. (Wiley \& Sons, New York) Ch1.

12 K. Kendall, in "Advanced Ceramic Materials" ed. R.J. Brook (Pergamon Press, Oxford, England, 1991, pp 365-369.

13 F.W.J. van Hattum, "A study of the mechanical properties of vapour grown carbon fibres and carbon fibre-thermoplastic composites, Ph.D. Thesis, 1999, University of Minho (Portugal).

14 R.P. Chartoff in Thermal Characterization of Polymeric Materials, Ed. E.A. Turi (Academic Press, San Diego, CA, U.S.A.), p.567 